\title{
Modeling of chemical and physical processes in reactive porous bed
} Jacek Kluska 1), Dariusz Kardaś 2)

\author{
The Szewalski Institute of Fluid Flow Machinery Polish Academy of Sciences \\ jkluska@imp.gda.pl ${ }^{1)}$, dk@imp.gda.pl ${ }^{2)}$
}

\begin{abstract}
This paper contains the description of pyrolysis and flow process through porous media taking into account kinetic rate coefficient and equilibrium state of pyrolysis. Mathematical equation of pyrolysis mechanism including kinetic rate coefficient and equilibrium rate of pyrolysis, and equations like mass, momentum and energy and flow through porous zone are presented. Considered model is based on a simple geometry based on downdraft gasifier and analysed basing on CFD calculation of pyrolysis and flow of volatiles. Numerical analysis of a gas flow in an open system with mass source is carried out by means of Fluent. Calculations of the chemical and physical processes in porous bed are performed for different porosity.
\end{abstract}

\section{Introduction}

Biomass, which contains mainly an organic substance, is a common renewable fuel in nature. The most popular kind of biomass is wood, which is available almost everywhere in the world. Biomass is utisied in combustion, gasification and pyrolysis processes. Gas produced in gasification process can be used for power generation in

. It is series of endothermic reactions which do not involve reactions with oxygen $[5,6]$. The products of pyrolysis are combustible gases, tar and char. In the presented work the pyrolysis process was described by means of one-step global mechanism (Fig.1). gas engine or directly combusted in boiler, which could lead to minimize the pollutants emission and $\mathrm{CO}_{2}$ reduction. These advantages generated considerable interest in the gasification of biomass. In a fixed bed gasifier biomass constitutes packed bed. The flow of volatiles and gases in a gasifier can be treated like a flow through porous media. Many investigations $[1,2,3,4]$ turn to CFD modeling thermochemical conversion of biomass in porous media because of the possibility of indepth look in high temperature and highly polluted region and comparing it with experimental data. CFD results could give informations on chemical and physical processes in packed bed and could help to understand experimental results. Finally, numerical solution could be used to optimize biomass gasification process.

\section{Biomass pyrolysis}

Pyrolysis referred to biomass gasification is the fundamental chemical process which occurs in gasifier but it is still not completely comprehensive.

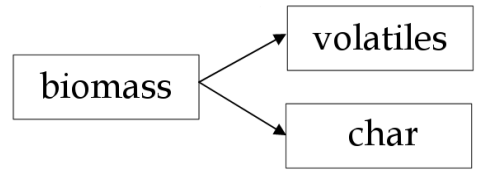

Fig.1. Sketch of one-step global mechanism of biomass pyrolysis. 
Taking into account that mass of biomass sample consists of mass of solid $m_{s}$ and gas phase $m_{g}$ :

$$
m=m_{s}+m_{g},
$$

where mass of solid $m_{s}$ consists of mass of volatiles $m_{\mathrm{v}}$ and residual mass $m_{r}$.

The change of solid phase during pyrolysis process can be defined as function of kinetic rate coefficient $k$, period of time $\Delta t$ and difference between solid mass of sample $m_{s}$ and residual mass $m_{r}$ :

$$
\Delta m_{s}=-k\left(m_{s}-m_{r}\right) \Delta t,
$$

where the reaction kinetic rate coefficient $k$ is usually expressed by Arrhenius law [1,4]:

$$
k=k_{0} e^{\left(-\frac{E}{R T}\right)},
$$

where: $E$ - energy activation $[\mathrm{kJ} / \mathrm{mol}]$,

$$
\begin{aligned}
& k_{0} \text { - constant, } \\
& R \text { - ideal gas constant }[\mathrm{J} / \mathrm{mol} \mathrm{K}] \\
& T \text { - temperature }[\mathrm{K}]
\end{aligned}
$$

According to literature kinetic rate coefficient can be also calculated by means of thermogravimetry measurements and given by $[7,8]$ :

$$
k=-\frac{\Delta m_{s}}{\left(m_{s}-m_{e}\right) \Delta t} .
$$

where $m_{e}$ in this case is a residual mass of solid phase in certain temperature for very slow heating rate. The rate of pyrolysis can be defined as $[9,10]$ :

$$
Z=\frac{m_{s}}{m_{s, 0}}
$$

where: $m_{\mathrm{s}}$ - mass of solid phase $[\mathrm{kg}]$,

$$
m_{s, 0} \text { - initial mass of solid phase }[\mathrm{kg}] \text {, }
$$

For the very low heating:

$$
\frac{\partial T}{\partial t} \rightarrow 0
$$

pyrolysis rate $Z$ can be function of temperature only and named as equilibrium rate of pyrolysis $Z_{e}(T)[9],[10]$. If the pyrolysis process is led with finite rate of heating the pyrolysis rate and equilibrium rate are different:

$$
Z(T) \neq Z_{e}(T)
$$

After simple calculations the change of pyrolysis rate can be expressed as a function of kinetic rate coefficient and deviation of pyrolysis rate $Z$ from equilibrium state:

$$
\frac{d Z}{d t}=-k\left(Z-Z_{e}\right)
$$

The relationship between pyrolysis rate $Z$ and equilibrium rate of pyrolysis $Z_{e}$ is presented in Fig2. 


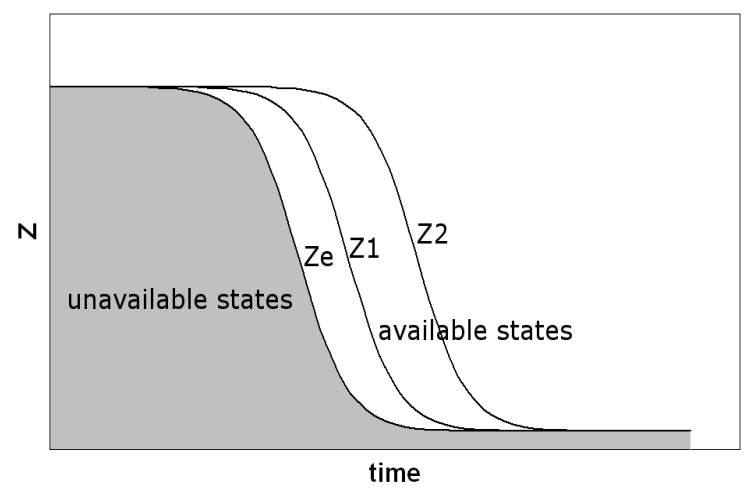

Fig. 2. Relationship between pyrolysis rate $Z$ and equilibrium rate of pyrolysis $\mathrm{Z}_{\mathrm{e}}$.

\section{Gasification process}

Biomass gasification consists of oxidation and reduction processes [5,6]. In the oxidation zone volatiles are burned with air to $\mathrm{CO}_{2}, \mathrm{CH}_{4}, \mathrm{CO}, \mathrm{H}_{2} \mathrm{O}$ and $\mathrm{H}_{2}$ in series of exothermic reactions. The heat obtained in exothermic reactions is used to release volatiles from biomass in pyrolysis process. In the reduction zone reactions between char and $\mathrm{CO}_{2}$ and $\mathrm{H}_{2} \mathrm{O}$ take place. All chemical reactions occurring in gasification process could be of volumetric or surface type. The main homogenous and heterogeneous reactions which can be found in literature $[1,6,12]$ are:

$$
\begin{gathered}
\mathrm{CH}_{4}+\mathrm{H}_{2} \mathrm{O}=\mathrm{CO}+3 \mathrm{H}_{2} \\
\mathrm{CO}+\mathrm{H}_{2} \mathrm{O}=\mathrm{CO}_{2}+\mathrm{H}_{2} \\
\mathrm{H}_{2}+\frac{1}{2} \mathrm{O}_{2}=\mathrm{H}_{2} \mathrm{O} \\
\mathrm{CH}_{4}+2 \mathrm{O}_{2}=\mathrm{CO}_{2}+2 \mathrm{H}_{2} \mathrm{O} \\
2 \mathrm{CO}+2 \mathrm{H}_{2}=\mathrm{CH}_{4}+\mathrm{CO}_{2} \\
\mathrm{C}+\mathrm{CO}_{2}=2 \mathrm{CO} \\
\mathrm{C}+\mathrm{O}_{2}=\mathrm{CO}_{2}
\end{gathered}
$$

$$
\begin{aligned}
& \mathrm{C}+\mathrm{H}_{2} \mathrm{O}=\mathrm{CO}+\mathrm{H}_{2}, \\
& 2 \mathrm{C}+\mathrm{O}_{2}=2 \mathrm{CO} .
\end{aligned}
$$

\section{Governing equations}

Physical and chemical processes in a reactive porous bed were described by the conservation laws of mass, momentum and energy for solid and gas phase and flow through porous media [1,11,14]. For the solid phase governing equations are given by:

$$
\begin{aligned}
\frac{\partial \varepsilon_{s} \rho_{s}}{\partial t}= & \nabla\left(\varepsilon_{s} \rho_{s} v_{s}\right)-S_{m} \\
\frac{\partial \varepsilon_{s} \rho_{s} U_{s}}{\partial t}+\nabla\left(\varepsilon_{s} \rho_{s} v_{s} U_{s}\right)= & -\alpha\left(T_{s}-T_{g}\right) \\
& +\nabla\left(\lambda \nabla T_{s}\right)-S_{U}
\end{aligned}
$$

And respectively for gas phase:

$$
\frac{\partial \varepsilon_{g} \rho_{g}}{\partial t}+\nabla\left(\varepsilon_{g} \rho_{g} v_{g}\right)=S_{m},
$$

$$
\frac{\partial \varepsilon_{g} \rho_{g} v_{g}}{\partial t}+\nabla\left(\varepsilon_{g} \rho_{g} v_{g} v_{g}\right)=-\nabla p+\nabla\left(\varepsilon_{g} \mu_{g} \nabla \mu_{g}\right)+S_{p},
$$

$$
\begin{aligned}
\frac{\partial \varepsilon_{g} \rho_{g} U_{g}}{\partial t}+\nabla\left(\varepsilon_{g} \rho_{g} v_{g} U_{g}\right) & =-p_{g} \nabla v_{g}+\alpha\left(T_{s}-T_{g}\right) \\
& +\nabla\left(\lambda \nabla T_{g}\right)+S_{U},
\end{aligned}
$$


where: $\mathcal{E}$ - porosity,

$$
\begin{aligned}
& v \text { - fluid velocity }[\mathrm{m} / \mathrm{s}], \\
& \rho \text { - density }\left[\mathrm{kg} / \mathrm{m}^{3}\right], \\
& S_{m} \text { - mass source term }\left[\mathrm{kg} / \mathrm{m}^{3} \mathrm{~s}\right], \\
& p \text { - pressure [Pa], } \\
& \mu \text { - viscosity [Pa s], } \\
& S_{p} \text { - Source term, resistance of solids to fluid } \\
& \quad \text { flow in a porous medium, } \\
& T \text { - temperature [K], } \\
& \lambda \text { - thermal conductivity [W/mK], } \\
& U \text { - internal energy [J], } \\
& \text { SU - heat source term [J] (due to heat release } \\
& \quad \text { during chemical reactions), } \\
& \mathrm{s}-\text { (lower subscript) - solid phase, } \\
& \mathrm{g} \text { - (lower subscript) - gas phase. }
\end{aligned}
$$

To better understand processes which occur in reactive porous bed presented model was simplified to one dimensional balance equations. Assuming that the solid phase is steady $v_{\mathrm{s}}=0$, so eq.(4.1),(4.2) do not contain convection term. The porosity of gas phase is defined as ratio of gases volume occupied by volatiles $V_{g}$ and the total volume $V$ :

$$
\varepsilon_{g}=\frac{V_{g}}{V} \text {. }
$$

Taking into account definition of volume fraction of solid, the relationship between porosity of gas and solid phase fulfill formula

$$
\varepsilon_{g}+\varepsilon_{s}=1
$$

Considering that the rate of pyrolysis (eq.(2.5)) in following form:

$$
Z=\frac{m_{s}}{m_{s, 0}}=\frac{\varepsilon_{s}}{\mathcal{E}_{s, 0}} \frac{\rho_{s}}{\rho_{s, 0}},
$$

where: $\rho_{s}$ - density of solid phase $\left[\mathrm{kg} / \mathrm{m}^{3}\right]$, $\rho_{s, 0}$ - initial density of solid phase $\left[\mathrm{kg} / \mathrm{m}^{3}\right]$,

the mass source in eq.(4.1) and (4.3) is given by equation:

$$
\varepsilon_{s, 0} \rho_{s, 0} \frac{d Z}{d t}=-k \varepsilon_{s, 0} \rho_{s, 0}\left(Z-Z_{e}\right)=S_{m} .
$$

Assuming that the pressure loss in the momentum equation (eq.(4.4)) is estimated by Darcy's law [3], which is based on the gas averaged velocity, the source term $S_{p}$ can be defined:

$$
S_{p}=\frac{\mu_{g} \varepsilon_{g}}{K} v_{g},
$$

where: $v_{g}$ - gas velocity $[\mathrm{m} / \mathrm{s}]$,

$$
\begin{aligned}
& K \text { - permeability }\left[\mathrm{m}^{2}\right], \\
& \mu_{g} \text { - gas viscosity [Pa s]. }
\end{aligned}
$$

The permeability can be also described [11] with regard to porosity $\varepsilon$ and particle diameter $D$ : 


$$
K=\frac{D^{2}}{150} \frac{\varepsilon^{3}}{(1-\varepsilon)^{2}}
$$

where: $D$ - biomass particle diameter [m],

$$
\mathcal{E} \text { - porosity of solid phase. }
$$

\section{Model description}

In presented work, the analyse of the rate of pyrolysis process, flow through porous media including gas temperature and velocity were presented. The geometry of CFD model which represents a simplified gasification chamber, is illustrated in Fig. 3. It is assumed that the chamber is filled by two porous materials in its right part. In general porosity in porous zone 1 was higher than in porous zone 2. It was assumed that beginning of the process cold gas (300K) flows into the chamber.

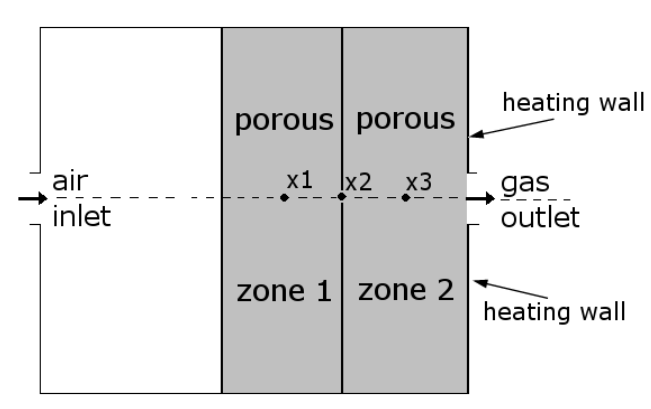

Fig.3. Reactor geometry and boundary conditions.

The heat is transported to the porous zones by heating wall, which was kept in $\mathrm{T}=1400 \mathrm{~K}$. Other walls was thermally stabilized in constant temperature $(300 \mathrm{~K})$. Air $(300 \mathrm{~K})$ enters inlet, flows through porous zones and cools material in zones 1 and 2. The heat is transported by conduction in right-left direction whereas opposite direction of heat is caused by convection of gases. The radiative heat transfer was described by P-1 model, which is the simplest formulation of the radiation model.

Described problems were treated as a nonstationary and resolved with usage of Ansys Fluent. For this case the mass, momentum and energy equations for gaseous phase, and mass and energy equations for solid phase were calculated. Mass source in mass balance equations (4.1) and (4.3) is dependent on temperature. Resistance of solids to fluid flow in a porous medium in momentum equation (4.4) is dependent on gas velocity and viscosity and zones permeability.

Referred to eq.(4.9) the change of pyrolysis rate depends on kinetic rate coefficient $k$, eviation of pyrolysis rate $Z$ and equilibrium rate $Z_{e}$. The equilibrium rate of pyrolysis $Z_{e}$ was set by thermogravimetric experimental results $[7,8]$ and approximated by temperature function $Z_{e}(T)$. Kinetic rate coefficient $k(T)$ was determined basic on thermogravimetric experimental results [7], which included residual mass of solid phase $m_{e}$ in certain temperature. Kinetic parameters were set at: $k_{0}=0.4 \quad \mathrm{~s}^{-1}$, $E / R=2850 \mathrm{~K}$. When the pyrolysis rate reached equilibrium state $Z=Z_{e}$ then pyrolysis process was stopped. The initial mass fraction of volatiles (ratio of volatiles mass $m_{v}$ and the total mass of solid phase $m_{s}$ ) was set at $Y_{v}=0.5$. The pyrolysis process was implemented by user defined function.

\section{Results and discussions}

Numerical analysis of pyrolysis process was calculated for two cases. In the first case (c1) porosity was set at $\varepsilon=0.8$ for fixed bed of biomass (porous zone 1 ) and $\varepsilon=0.4$ for fixed bed of char (porous zone 2). The diameter of biomass particle was set at $d=8 \mathrm{~cm}$. In the second case ( $c 2$ ) porosity was set at $\varepsilon=0.5$ for 
fixed bed of biomass and $\varepsilon=0.3$ for fixed bed of char and the diameter of biomass particle was set at $d=2 \mathrm{~cm}$.

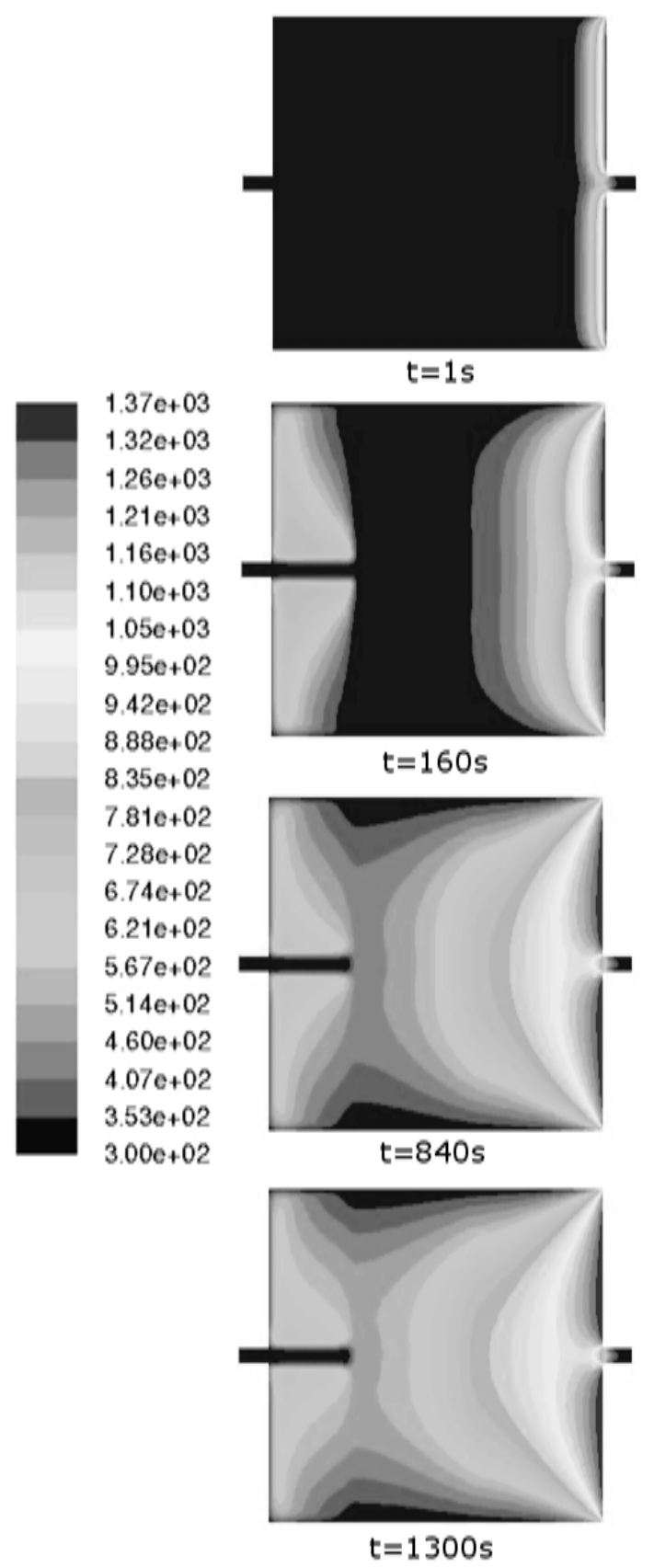

Fig.4. Contours of static temperature $[\mathrm{K}]$ for case 1 for the particular time steps.

Figure 4 illustrates the temperature front of pyrolysis. The fixed bed become heated very slow due to porosity. On the left part of chamber filled by gases the temperature increased faster than in porous zones which is caused by gas heating by radiation from heating wall. A distribution of temperature for first case is illustrated in Fig.5. for the three representative points: $x 1$-porous zone 1 , x2- midpoint, $x 3$ - porous zone 2 (Fig.3).

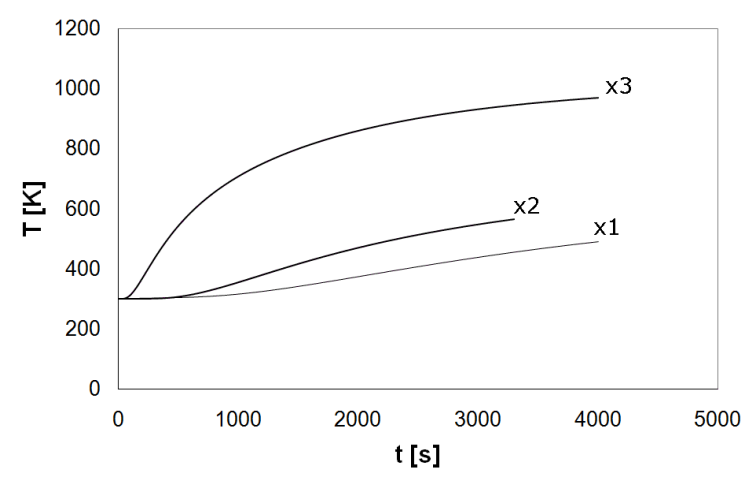

Fig.5. The temperature distribution of pyrolysis in porous zones.

Figure 6 illustrates the rate of pyrolysis in porous zones for case1. The distribution of temperature reached a maximum value at point $x 3$ and the pyrolysis rate decreased to minimum value $Z=0.05$, which represented equilibrium rate $Z_{e}$ for maximum temperature, in $t=950 \mathrm{~s}$. In this point the process of devolatilization can be treated as finished. For points $x 1$ and $x 2$ temperature did not reach maximum value so the rate of pyrolysis changed insignificantly. The deviation of pyrolysis rate $Z$ from equilibrium rate $Z_{e}$ in point $x 3$ is presented in Fig.7. 


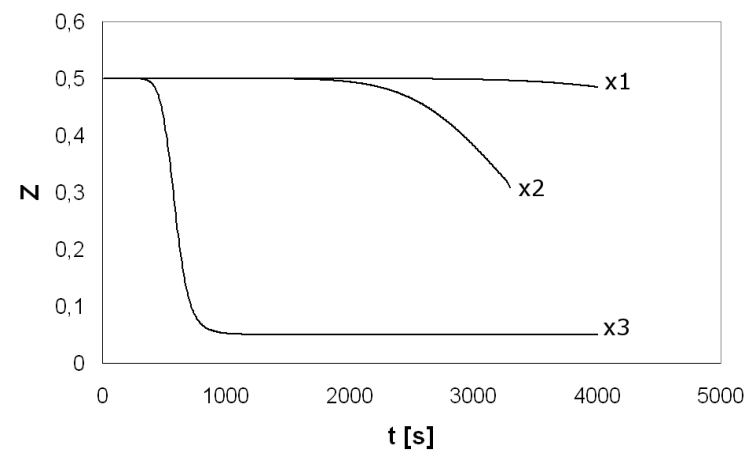

Fig.6. The rate of pyrolysis in temperature function for case 1.

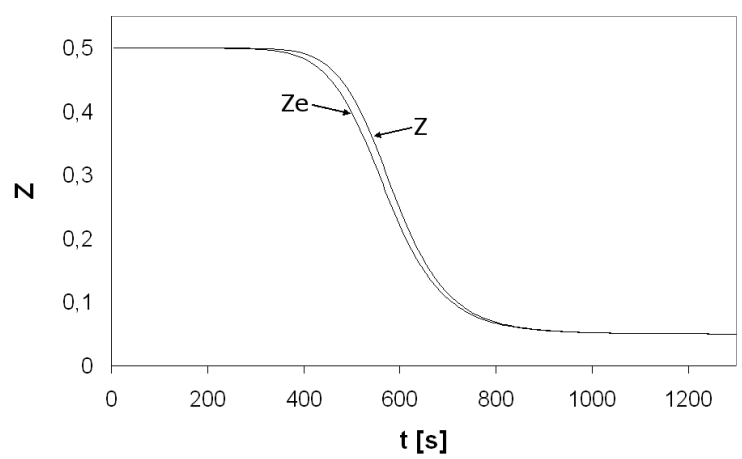

Fig.7. The pyrolysis rate $Z$ and equilibrium rate of pyrolysis $Z_{e}$ in temperature function at point x3 for case1.

Comparison of two cases $(\mathrm{c} 1, \mathrm{c} 2)$ of zones temperature for points $\mathrm{x} 1, \mathrm{x} 2$ and $\mathrm{x} 3$ are presented in Figs.8-10. Process of heat propagation was slightly different in each case. The temperature distribution for case 1 was faster in each point which was caused by higher porosity than in case 2 .

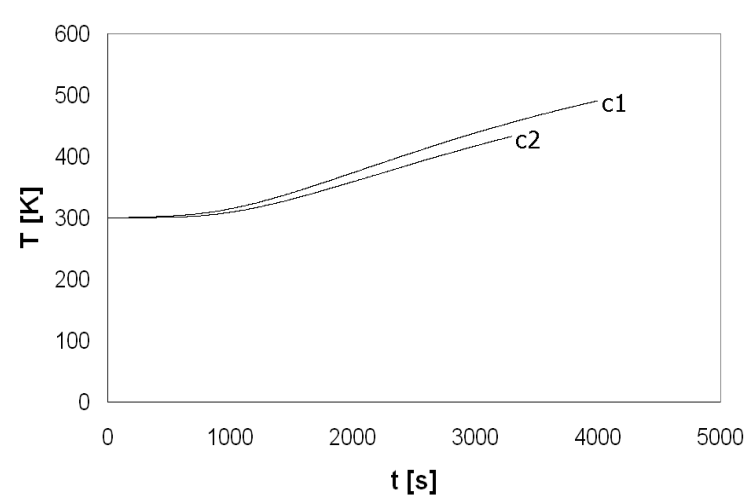

Fig.8. Comparison of temperature distribution at point $x 1$ for two cases of porous zones in fixed bed.

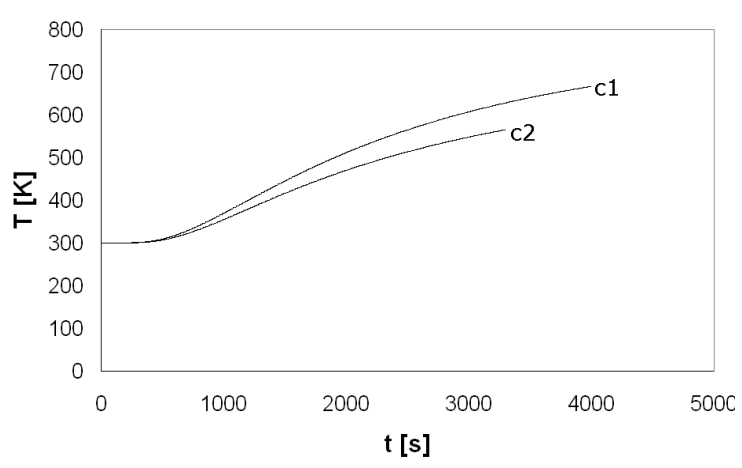

Fig.9. Comparison of temperature distribution at point $x 2$ for two cases of porous zones in fixed bed.

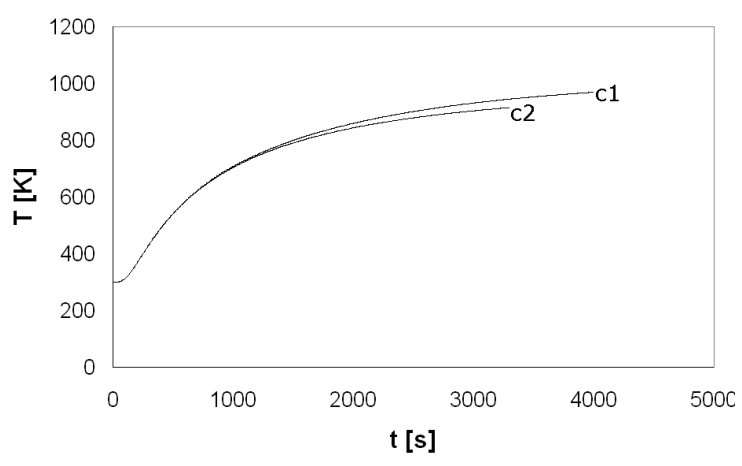

Fig.10. Comparison of temperature distribution at point $x 3$ for three cases of porous zones in fixed bed.

The differences in distribution of temperature for various porosity provide to the different rate of pyrolysis which is illustrated for point $x 2$ in Fig.11. The pyrolysis was faster for case $\mathrm{c} 1$ due to lower 
heat capacity of porous bed in this case and better transport of radiation.

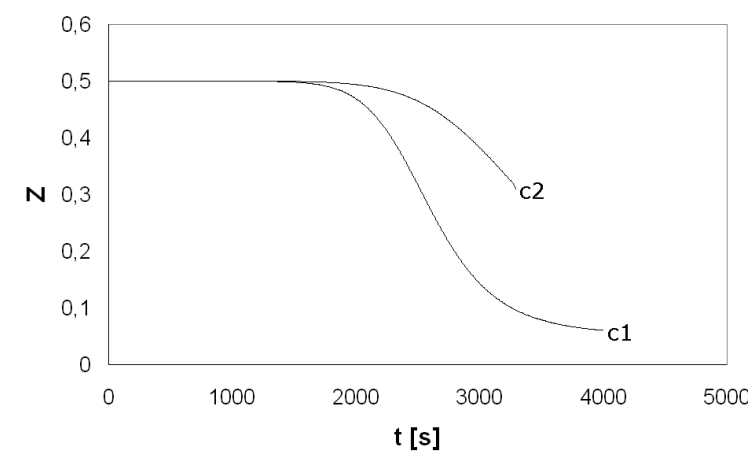

Fig.11. The rate of pyrolysis at point $x 2$ for two cases of porous zones in fixed bed.

The analysis of flow through porous media showed different influence of porosity on magnitude of gas $x$-velocity which is shown for points $x 2$ and $x 3$ in Fig.12 and Fig.13. For case 1 the $x$-velocity was higher than for case 2 in points $x 2$ and $x 3$ due to higher zone porosity and (with regard to eq.(4.10) and (4.11)) higher permeability.

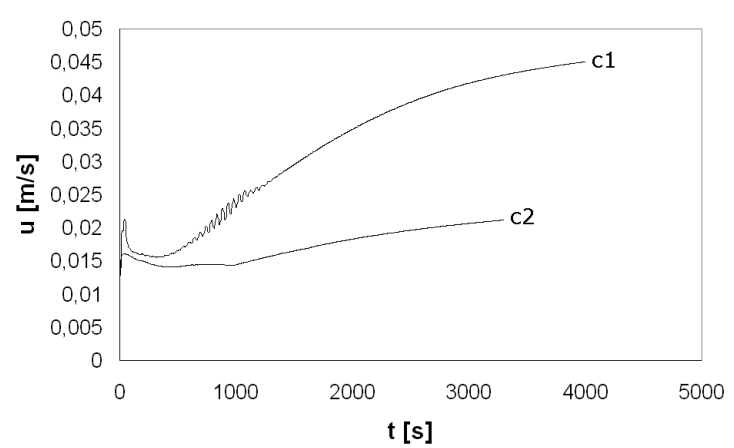

Fig.12. Comparison of $x$-velocity at point $x 2$ for two cases against the time.

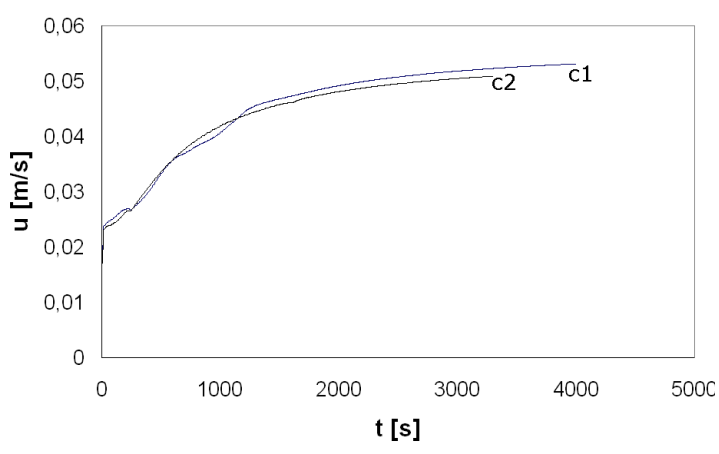

Fig.13. Comparison of $x$-velocity at point $x 3$ for two cases against the time.

For each case increase of gas velocity during flow through porous zones was observed (Fig.14 and 15). It was caused by increase of gas temperature and production of additional gases in pyrolysis process.

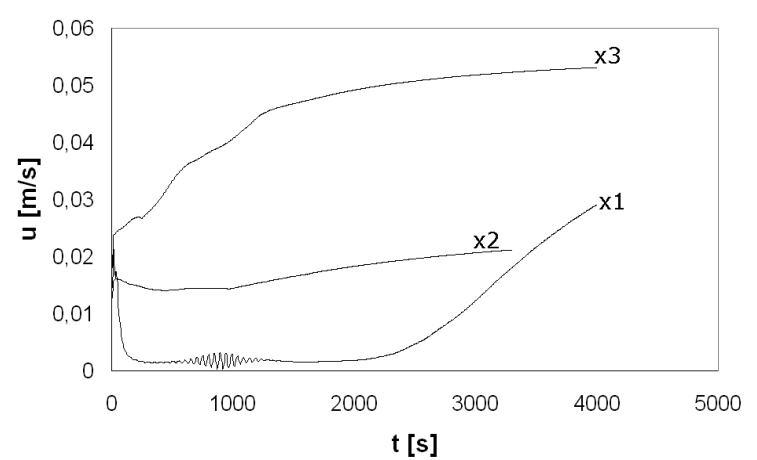

Fig.14. Comparison of $x$-velocity at points $x 1, x 2$ and $\mathrm{x} 3$ for case1.

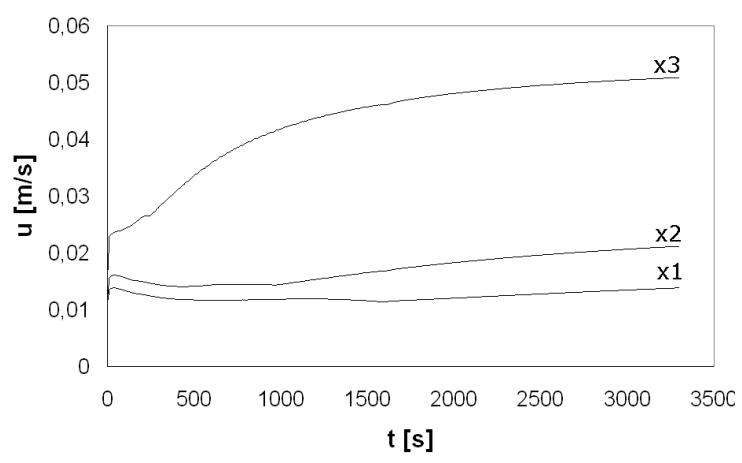

Fig.15. Comparison of $x$-velocity at points $x 1, x 2$ and $\mathrm{x} 3$ for case2. 


\section{Summary}

A devolatilization model in porous medium has been developed. The pyrolysis mechanism including kinetic rate coefficient $k$ and equilibrium state of pyrolysis $Z_{e}$ were presented. Presented work showed that user defined function makes the modeling of pyrolysis process in CFD calculations possible. The change rate of pyrolysis can be described by function dependent on kinetic rate coefficient $k$ and deviation of pyrolysis rate from equilibrium state $Z-Z e$.

In consequence distribution of temperature in porous zones, the temperature front of pyrolysis was generated and the rate of pyrolysis was variable in fixed bed. In this case different gas velocity was observed. Increase of gas velocity was caused by heating of porous bed and released volatiles - source terms in mass and energy equations.

In summary the numerical modeling of chemical and physical processes in reactive porous bed can be use to predict and analyse the biomass pyrolysis.

\section{Acknowledgements}

The investigations presented in this paper have been obtained within the Research Project No. POIG.01.01.02-00-016/08. Model agroenergy complexes as an example of distributed cogeneration based on a local renewable energy sources.

\section{REFERENCES}

[1] Yiqun Wang and Lifeng Yan, CFD Studies on Biomass Thermochemical Conversion, Int. J. Mol. Sci. 2008, 9, 1108-1130.

[2]Noorhelinahani Abu Bakar, Downdraft Gasifier Using Computational Fluid Dynamics SoftwareFluent, A thesis submitted for the deegre of Master of Science 2003.
[3] C. Brucha, B. Petersb, T. Nussba, Modelling wood combustion under fixed bed conditions, Fuel 82 (2003) 729-738.

[4] A.A. Rostami, M.R. Hajaligol, S.E. Wrenn, A biomass pyrolysis sub-model for CFD applications, Fuel 83 (2004) 1519-1525.

[5]S. Porada, The reactions of formation of selected gas products during coal pyrolysis, Fuel 83 (2004) 11911196.

[6]A. K. Rajvanshi, Biomass Gasification, Alternative Energy in Agriculture (1986) 83-102.

[7] J. Guo, A. C., Lua, Kinetic study on pyrolysis of extracted oil palm fiber. Isothermal and non isothermal conditions , Journal of Thermal Analysis and Calorimetry, Vol. 59 (2000) 763.774.

[8]O. Senneca, Kinetics of pyrolysis, combustion and gasification of three biomass, Fuel Processing Technology 88 (2007) 87-97.

[9]D. Kardaś, Model szybkości pirolizy węgla i biomasy z uwzględnieniem nierównowagi termodynamicznej układu, Polska Akademia Nauk, Instytut Maszyn Przepływowych w Gdańsku, Nr arch. 227/2010. 10]S. Postrzednik, Ciepto odgazowania paliw statychmetoda określania, podstawowe własności, Karbo, Energiochemia, Ekologia, KSG, 39(9):220-228,1994. [11]FLUENT 6.3 User's Guide, September 2006.

[12]N. Prakash , T. Karunanithi. Kinetic Modeling in Biomass Pyrolysis - A Review, Journal of Applied Sciences Research, 4(12): 1627-1636, 2008. [13]H. Yang, R. Yan, H. Chen, D. Lee, C. Zheng, Characteristics of hemicellulose, cellulose and lignin pyrolysis, Fuel 86 (2007) 1781-1788.

[14]Yang Y.B., Ch. Ryu, A. Khor, V.N. Sharifi, J. Swithenbank, Fuel size effect on pinewood combustion in a packed bed, Fuel 84 (2005) 2026-2038. 